\title{
Revisión clínica y epidemiológica de la leishmaniasis tegumentaria en una región central del Perú
}

W. Samir Cubas ${ }^{1,2}$, Dercy Centeno-Leguía, Kovy Arteaga-Livias y Edwin Depaz-López,2

\section{Clinical and epidemiological review of tegumentary leishmaniasis in a central region of Peru}

Background: Leishmaniasis is an infectious disease with a high prevalence and worldwide incidence, causing around 70,000 deaths per year. Aim: To determine the clinical and epidemiological profile of cases of tegumentary leishmaniasis in the Province of Ambo, Department of Huánuco, Peru, during the period from 2000 to 2017. Methods: A descriptive, observational, retrospective and cross-sectional study was conducted population base. Results: We identified 1,312 confirmed cases of tegumentary leishmaniasis, the cutaneous clinical form had a higher prevalence compared to mucocutaneous (83.2 vs $16.7 \%)$. The infection in the masculine gender had greater predominance in front of the feminine one ( $53.3 \mathrm{vs} 46.6 \%)$. The highest reported annual incidence rate was $412.67 / 100,000$ inhabitants during the year 2000 while the lowest registered was in 2015 with $24.45 / 100,000$ inhabitants. The Ambo district was the most affected in the clinical cutaneous form (43.1\%) as mucocutaneous $(38.2 \%)$ of the disease. The main diagnostic method for both clinical forms was microscopy $(82.0 \mathrm{vs} 81.0 \%)$ and the most used therapy was sodium stibogluconate ( $85.6 \mathrm{vs} 78.6 \%$ ). Failure to treatment was reported in $25 \%$ for both presentations clinics. Conclusions: The eco-environmental conditions of this area of Peru have allowed the establishment of tegumentary leishmaniasis; showing trends similar to world literature. However, more research is needed to explain the epidemiological behavior of this disease that allows the creation of epidemiological prediction models for timely and adequate control.

Keywords: Infectious diseases; neglected tropical diseases; leishmaniasis; epidemiological profile; Huanuco, Peru.

Palabras clave: Enfermedades infecciosas; enfermedades tropicales desatendidas; leishmaniasis; perfil epidemiológico; Huánuco, Perú.

\section{Introducción}

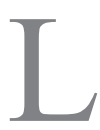

a leishmaniasis es una de las enfermedades infecciosas emergentes y re-emergentes de gran importancia a nivel mundial, causada por protozoos del género Leishmania (Familia Trypanosomatidae) y transmitida por la picadura del díptero hematófago hembra infectado del género Phlebotomus (Europa, África y Asia) y Lutzomyia (América) ${ }^{1}$. Existe una gran diversidad de mamíferos salvajes y domésticos que actúan como reservorios (cánidos, roedores y marsupiales) y son considerados como fuentes de transmisión al hombre; aunque en las formas antroponóticas, prevalentes en la India y Sudán, el hombre es el principal reservorio de la enfermedad ${ }^{2}$. La prevalencia mundial de leishmaniasis se estima en 12 millones de casos, es causante de alrededor de 70.000 muertes cada año y cuenta con una incidencia anual de 1,3 millones, cifras que van en aumento ${ }^{3,5}$. Esta enfermedad es endémica en las Américas, la cuenca del Mediterráneo y Asia occidental (desde el Medio Oriente hasta Asia Central) con 70 a $75 \%$ de los casos en 10 países:
Afganistán, Argelia, Colombia, Brasil, Irán, Siria, Etiopía, Sudán del Norte, Costa Rica y Perú ${ }^{6,7}$.

Las formas clínicas leishmaniasis son: cutánea, muco-cutánea y visceral; la gravedad de la infección dependerá de factores como la especie del protozoo y el tipo de respuesta inmune 5 . En la actualidad, el Perú cuenta con una tasa de incidencia anual de leishmaniasis de $13,4 / 100.000$ habts. y más de $60 \%$ de los casos reportados corresponde a los departamentos de Huánuco, Cuzco, Madre de Dios, Cajamarca, Junín, Amazonas y San Martín ${ }^{8}$. En el Perú, la leishmaniasis se presenta de dos formas clínicas: la cutánea andina o "uta", causada principalmente por Leishmania peruviana, endémica de los valles occidentales e interandinos ubicados entre los 800 y 3.000 msnm, y la selvática cutánea y muco-cutánea o "espundia" causada generalmente por Leishmania braziliensis en valles orientales de la selva alta y toda la llanura amazónica ubicadas por debajo de los 1.800 $\mathrm{msnm}^{9,10}$. En el Perú, los departamentos de Madre de Dios y Huánuco reportaron hace más de 25 años las tasas de incidencia anual más elevadas (490 vs $175 / 100.000$
'Área de Medicina del Centro de Salud de Ambo. Huánuco, Perú. ${ }^{2}$ MicroRed de Salud de Ambo, Huánuco, Perú.

${ }^{3}$ Escuela Profesional de Medicina Humana, Universidad Nacional de San Cristóbal de Huamanga, Ayacucho, Perú.

${ }^{4}$ Facultad de Medicina, Universidad Nacional Hermilio Valdizán, Huánuco, Perú.

Los autores declaran no tener conflictos de interés.

La presente investigación no ha recibido financiamiento externo a los autores.

Recibido: 11 de septiembre de 2018

Aceptado: 9 de mayo de 2019

Correspondencia a:

Wildor Samir Cubas Llalle wsamircubas@gmail.com 
habts.), siendo la Provincia de Ambo, Departamento de Huánuco, una de las zonas más hiperendémicas de la región $(1.158,34 / 100.000 \text { habts. })^{10,11}$. Las políticas y estrategias de control epidemiológico llevadas a cabo a lo largo de los años por el Ministerio de Salud lograron disminuir significativamente las altas tasas de incidencia y prevalencia de leishmaniasis en el país.

Debido a la importancia epidemiológica y el impacto de leishmaniasis en la salud, son importantes las investigaciones que reflejen el comportamiento epidemiológico de esta enfermedad en el transcurso de estos últimos años, con la finalidad de crear modelos de predicción epidemiológica que permitan tomar medidas de control oportunas y adecuadas.

El presente estudio tiene como objetivo determinar el perfil clínico y epidemiológico de los casos de leishmaniasis tegumentaria en la Provincia de Ambo, Departamento de Huánuco, Perú diagnosticados durante el período del 2000 al 2017.

\section{Métodos}

\section{Diseño y población de estudio}

Se realizó un estudio descriptivo, observacional, retrospectivo, transversal, de base poblacional en la Provincia de Ambo, Departamento de Huánuco, Perú $\left(10^{\circ} 08^{\prime} 00^{\prime \prime} \mathrm{S}\right.$, $76^{\circ} 12^{\prime} 00^{\prime \prime} \mathrm{O}$, población $\sim 58,000$ ) (Figura 1). La provincia se encuentra a $2.064 \mathrm{msnm}$, está conformada por ocho distritos (Ambo, Cayna, Huacar, Colpas, San Francisco, San Rafael, Conchamarca y Tomay Kichwa) y cuenta con una extensión de $1.581,00 \mathrm{~km}^{2}$. Se encuentra ubicada en el delta de los ríos Huallaga y Huertas, la temperatura media es superior a $25^{\circ} \mathrm{C}$ y la humedad relativa varía entre 60 y $95 \%$, con precipitaciones pluviales abundantes de enero a marzo; el relieve es accidentado y el tipo de vegetación predominante son las gramíneas, siendo la agricultura la actividad económica más importante, seguida por la ganadería.

\section{Tamaño de muestra poblacional}

Se evaluaron las fichas y reportes epidemiológicos de todos los casos diagnosticados de leishmaniasis por observación de amastigotas de Leishmania sp en improntas de material obtenido de las lesiones de la piel y mucosas a través del examen microscópico directo, de acuerdo al Manual para el Diagnóstico, Tratamiento y Control de la Leishmaniasis del MINSA, entre los años 2000 y 2017 en la Provincia de Ambo. Se excluyeron los casos que no cumplieran con este requisito.

\section{Recolección de datos y variables del estudio}

Se utilizó como fuente primaria de información los reportes y fichas epidemiológicas otorgadas por las Microredes de Salud y la Oficina General de Epidemiología de la Dirección Regional de Salud del Departamento de Huánuco; se seleccionaron las fichas con diagnóstico de leishmaniasis de la provincia de Ambo, según el orden cronológico y en segunda instancia se procedió al levantamiento de datos. Las variables de edad, género, año de diagnóstico y presentación clínica de la enfermedad fueron tomadas directamente de los reportes epidemiológicos anuales; mientras que variables ámbito laboral, procedencia, distribución de las lesiones, tipo
Figura 1. Ubicación del área de estudio de la leishmaniasis tegumentaria en el Perú del 2000 al 2017.

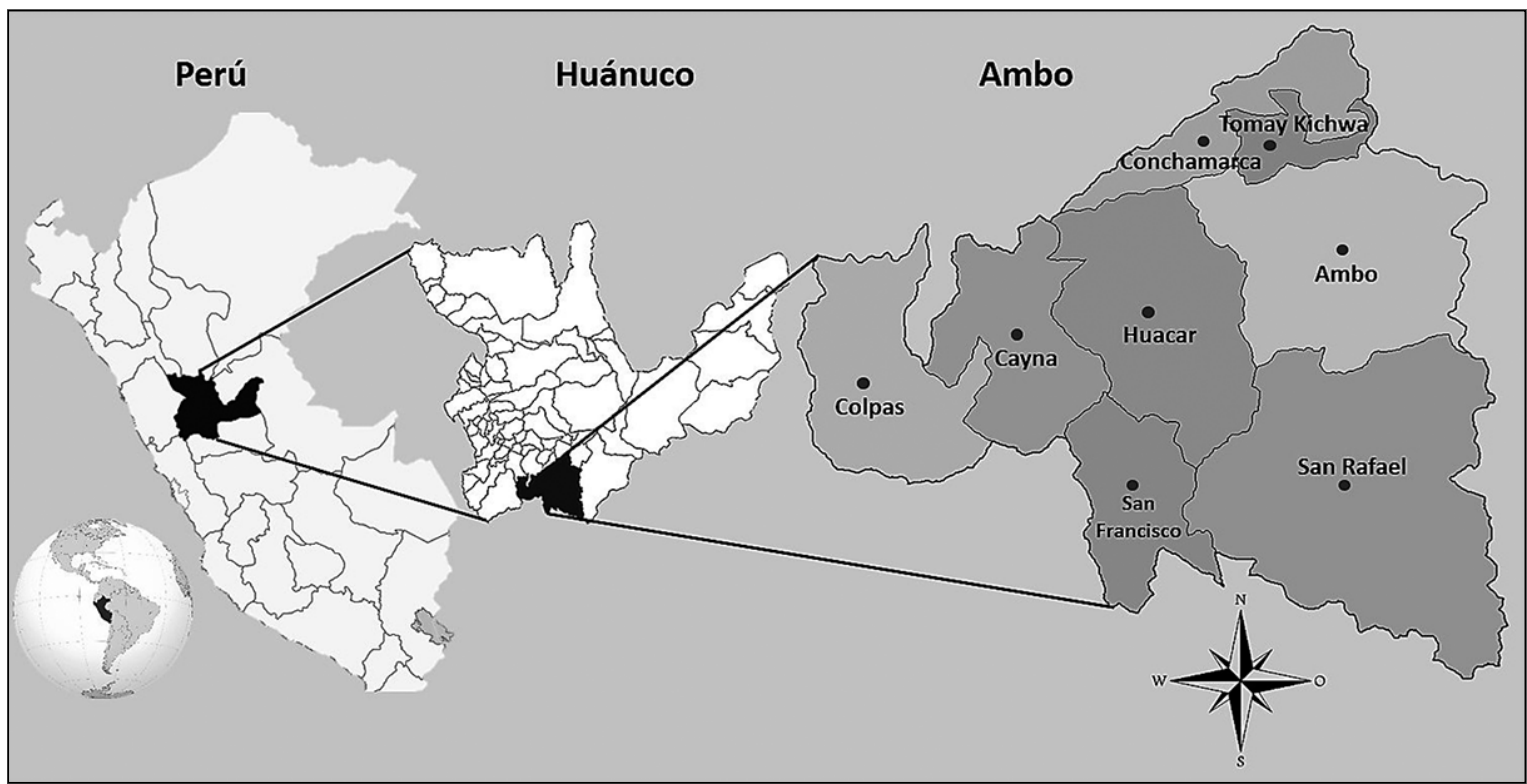

Rev Chilena Infectol 2019; 36 (6): 707-715 
de diagnóstico, tratamiento administrado y resultado del caso fueron obtenidas en forma indirecta de las fichas epidemiológicas. Los datos fueron doble digitados y revisados mediante una lista de verificación.

\section{Análisis estadístico}

Se realizó un análisis descriptivo de las características clínico-epidemiológicas basales de la muestra de estudio. Se calculó la incidencia y prevalencia de la leishmaniasis tegumentaria en la Provincia de Ambo con las variables edad, género, ámbito laboral, procedencia, distribución de las lesiones, tipo de diagnóstico, tratamiento administrado y resultado del caso; para ello, en todos los casos el análisis de los datos se realizó utilizando el programa estadístico STATA MP v13 y Microsoft Excel v13.

\section{Aspectos éticos}

La Dirección Regional de Salud del Departamento de Huánuco y el Comité de Ética del Hospital Nacional Docente Madre Niño "San Bartolomé” (RCEI-40), Lima, Perú, revisaron y aprobaron el protocolo de este estudio. Se respetó la confidencialidad de la información y no fue necesario el uso del consentimiento informado porque los datos se obtuvieron de forma retrospectiva e indirecta.

\section{Resultados}

En la Provincia de Ambo, durante el período 20002017, se registraron 1,312 casos de leishmaniasis tegu- mentaria, presentando la forma clínica cutánea (1.092 casos; 83,2\%) la mayor cantidad de casos, seguida de la leishmaniosis muco-cutánea (220 casos; 16,7\%) (Figura 2). En relación al sexo, se encontró un predominio de la forma clínica tegumentaria en el género masculino $(53,3 \%)$ en comparación al femenino $(46,6 \%)$; de acuerdo a la presentación clínica, el género femenino fue superior en la forma cutánea $(50,1 \%)$ mientras que el masculino en la muco-cutánea $(56,8 \%)$. La edad promedio de todos los casos reportados fue de $23 \pm 10$ años (Rango: 1-97 años) (Figura 3). Al caracterizar los resultados según la procedencia, se identificó que el Distrito de Ambo cuenta con la mayor cantidad de casos reportados por leishmaniasis tegumentaria (40,6\%) a lo largo de últimos 18 años. En dicho distrito, se evidenció que la forma clínica cutánea $(43,1 \%)$ de la enfermedad tiene una mayor afectación que la muco-cutánea $(38,2 \%)$; por otro lado, el segundo distrito donde se reportó una gran cantidad de casos por leishmaniasis tegumentaria fue Huacar $(24,5 \%)$, siendo la afectación clínica muco-cutánea $(26,8 \%)$ mayor que la cutánea $(22,2 \%)$. Se determinó que $42,8 \%$ de los casos reportados por leishmaniasis tegumentaria correspondían a pacientes desempleados; en esta categoría se incluyó a la población económicamente inactiva como el caso de amas de casa, pacientes de la niñez y senectud. En el estrato de la población económicamente activa, la leishmaniasis tegumentaria afectó considerablemente a pacientes dedicados al rubro de la agricultura $(33,8 \%)$, ganadería $(12 \%)$ y con una menor prevalencia en profesionales $(11,3 \%)$ (Tabla 1).

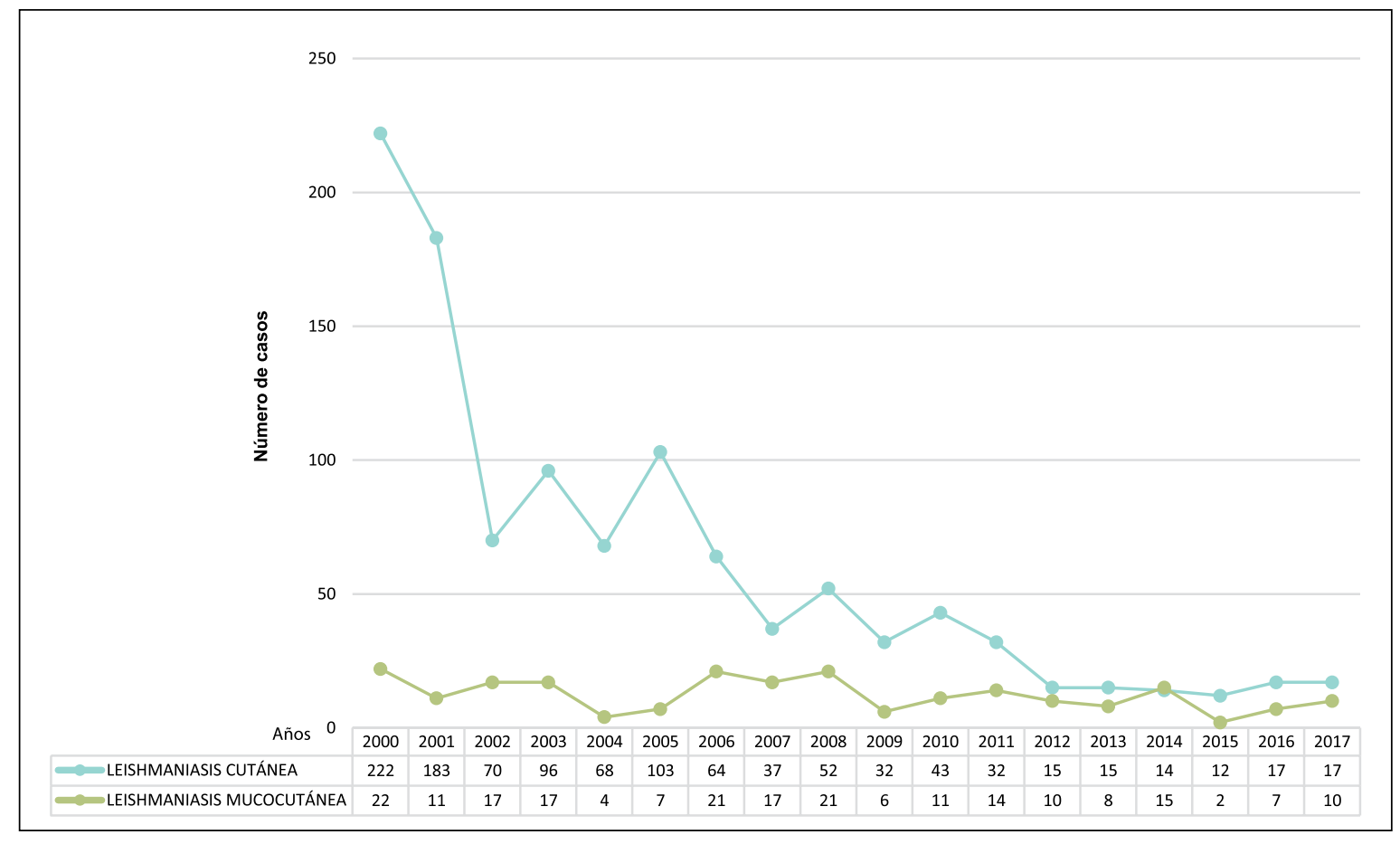

Rev Chilena Infectol 2019; 36 (6): 707-715
Figura 2. Evolución epidemiológica anual de la de la leishmaniasis tegumentaria en la Provincia de Ambo, Huánuco, Perú. 2000-2017. 
Figura 3. Distribución gráfica de los casos de leishmaniasis tegumentaria por grupos de edad en la Provincia de Ambo, Huánuco, Perú. 2000-2017.

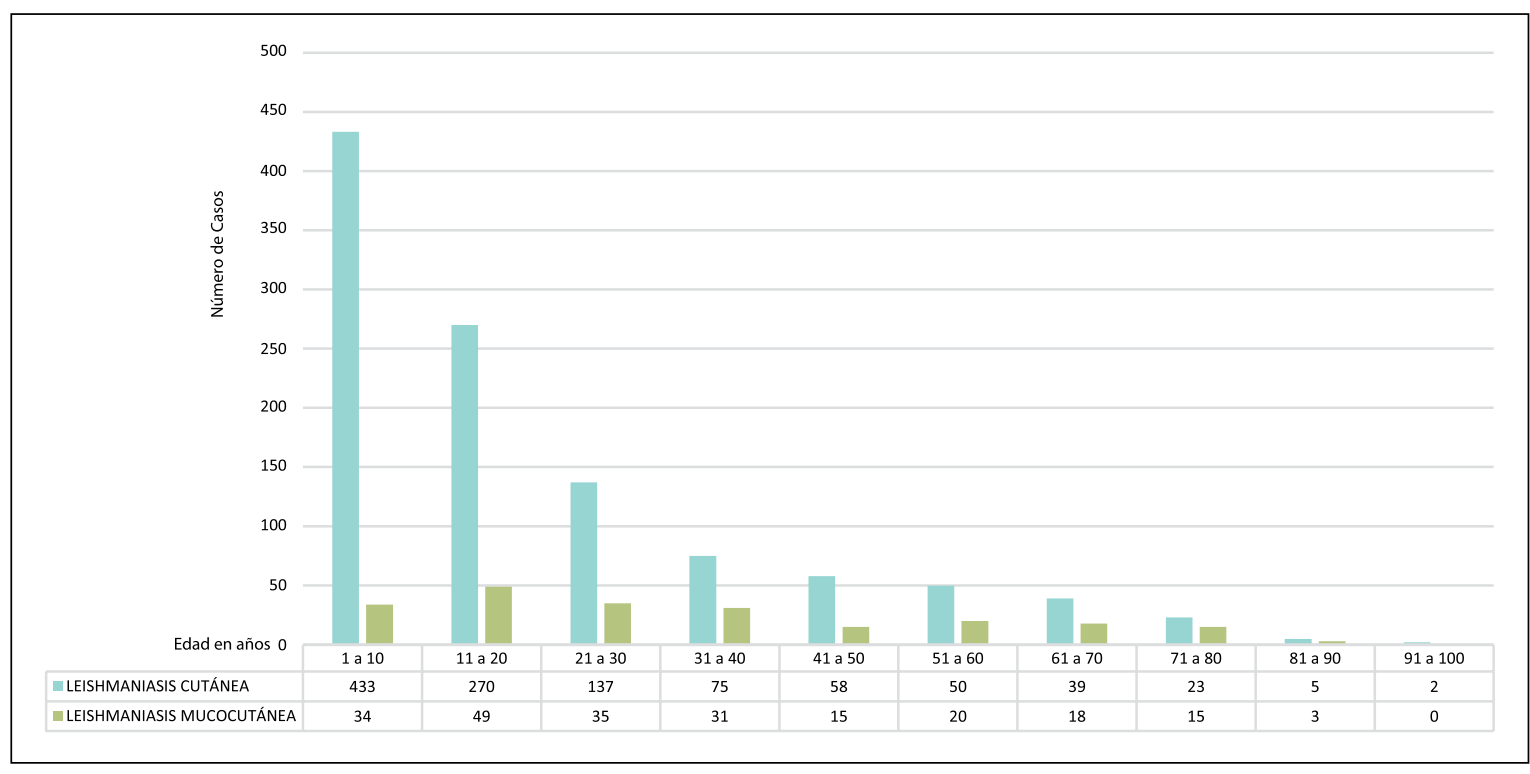

El mayor número de casos reportados de leishmaniasis tegumentaria corresponde al año 2000 (244 casos), con una tasa de incidencia anual de 412.67/100.000 habts. y una prevalencia anual de $0,41 \%$ de la población (Figura 4). Durante el año 2015, se obtuvo la más baja tasa de incidencia $(24,45 / 100.000$ habts. ) y prevalencia anual $(0,02 \%$ de la población) de leishmaniasis tegumentaria (Tabla 2). Se registró una mayor prevalencia en pacientes entre los 1 a 10 años seguida de 11 a 20 años $(35,5$ vs 24,3$)$. En todos los casos, la leishmaniasis cutánea $(83,2 \%)$ tuvo mayor prevalencia que la mucocutánea $(16,7 \%)$ (Figura 3). En cuanto a la distribución corporal de las lesiones producidas por la infección, la cara fue el lugar que reportó la mayor cantidad de lesiones por leishmaniasis cutánea seguida de los miembros superiores e inferiores $(51,1$ vs 26,6 vs $10,8 \%$ ); mientras que en la leishmaniasis muco-cutánea el total de lesiones (100\%) incluyó a la mucosa buco-nasofaríngea. El principal método diagnóstico para la forma clínica cutánea como muco-cutánea fue el examen directo por microscopía $(82,0$ vs $81,0 \%)$. La inmunofluorescencia indirecta (IFI) estuvo limitada a un bajo número de casos de leishmaniasis cutánea $(18,0 \%)$ y muco-cutánea $(19,0 \%)$. El tratamiento administrado a la leishmaniasis incluyó el estibogluconato de sodio y el antimoniato de $\mathrm{N}$-metilglucamina (Glucantime ${ }^{\circledR}$ ) en su forma cutánea ( 85,6 vs $14,4 \%)$ y muco-cutánea $(78,6$ vs $21,4 \%)$. Se obtuvieron tasas de curación de la presentación cutánea y muco-cutánea $(56,9$ vs $64,5 \%)$ y una persistencia de la enfermedad en $25,0 \%$ de ambas formas clínicas. Se reportaron cifras de abandono del tratamiento de las formas clínicas cutánea y muco-cutánea ( 18,1 vs $10,5 \%)$ (Tabla 1). 


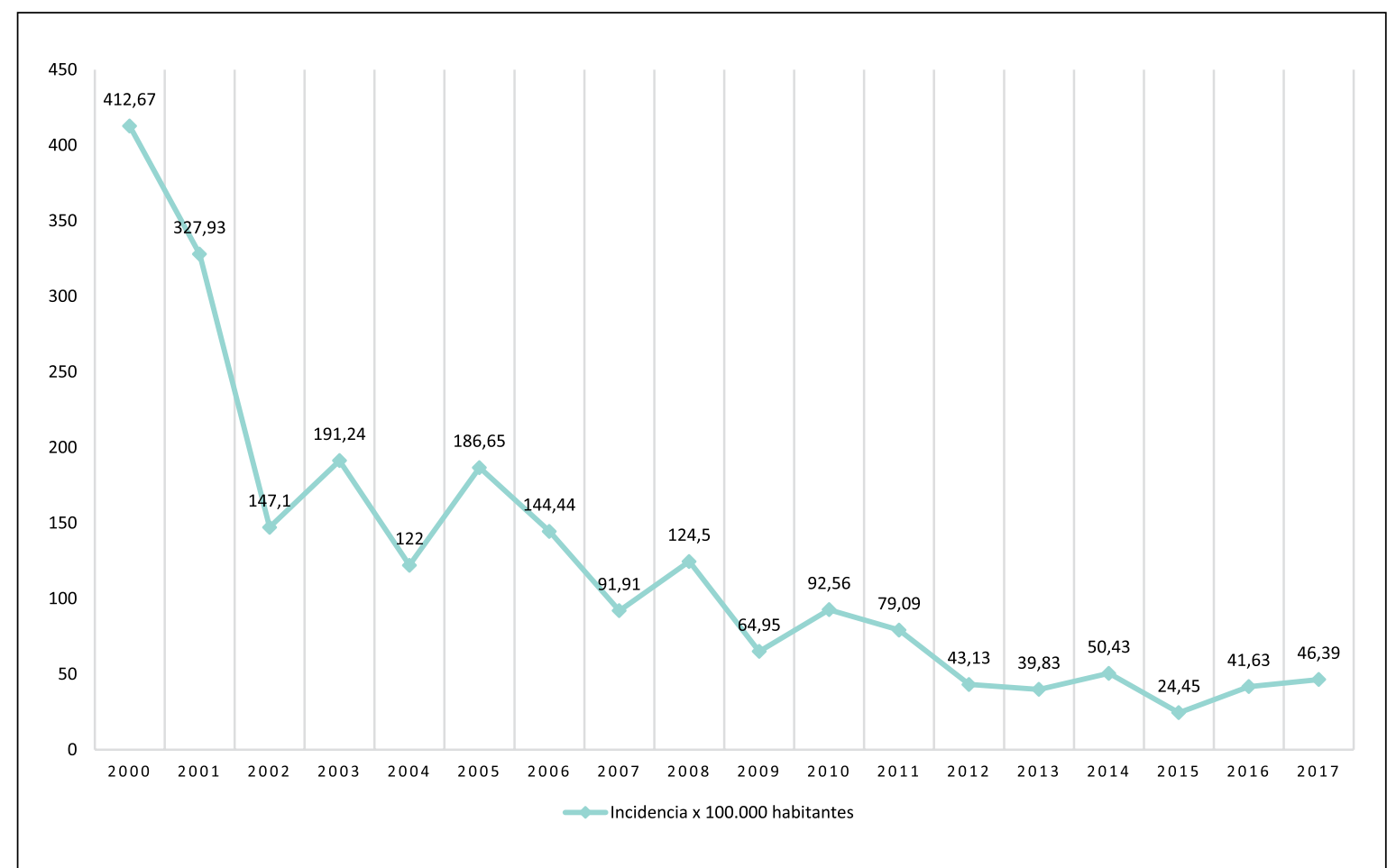

Figura 4. Distribución gráfica de tasa de incidencia anual de leishmaniasis tegumentaria en la Provincia de Ambo, Huánuco, Perú. 2000-2017.

Tabla 2. Números de casos, Incidencia y prevalencia de la leishmaniasis tegumentaria en la Provincia de Ambo, Huánuco, Perú. 2000-2017

\begin{tabular}{|c|c|c|c|c|c|}
\hline Años & Población aproximada & $\mathrm{n}$ de casos & Incidencia anual & $\begin{array}{c}\text { Tasa de incidencia anual } \\
(x 100.000 \text { habts. })\end{array}$ & $\begin{array}{c}\text { Prevalencia anual } \\
\%\end{array}$ \\
\hline 2000 & 59.126 & 244 & 0,0041 & 412,67 & 0,41 \\
\hline 2001 & 59.158 & 194 & 0,0032 & 327,93 & 0,32 \\
\hline 2002 & 59.141 & 87 & 0,0014 & 147,10 & 0,14 \\
\hline 2003 & 59.088 & 113 & 0,0019 & 191,24 & 0,19 \\
\hline 2004 & 59.013 & 72 & 0,0012 & 122,00 & 0,12 \\
\hline 2005 & 58.932 & 110 & 0,0018 & 186,65 & 0,18 \\
\hline 2006 & 58.847 & 85 & 0,0014 & 144,44 & 0,14 \\
\hline 2007 & 58.749 & 54 & 0,0009 & 91,91 & 0,09 \\
\hline 2008 & 58.634 & 73 & 0,0012 & 124,50 & 0,12 \\
\hline 2009 & 58.499 & 38 & 0,0006 & 64,95 & 0,06 \\
\hline 2010 & 58.339 & 54 & 0,0009 & 92,56 & 0,09 \\
\hline 2011 & 58.158 & 46 & 0,0007 & 79,09 & 0,07 \\
\hline 2012 & 57.957 & 25 & 0,0004 & 43,13 & 0,04 \\
\hline 2013 & 57.737 & 23 & 0,0003 & 39,83 & 0,03 \\
\hline 2014 & 57.498 & 29 & 0,0005 & 50,43 & 0,05 \\
\hline 2015 & 57.239 & 14 & 0,0002 & 24,45 & 0,02 \\
\hline 2016 & 57.644 & 24 & 0,0004 & 41,63 & 0,04 \\
\hline 2017 & 58.200 & 27 & 0,0004 & 46,39 & 0,04 \\
\hline
\end{tabular}




\section{Discusión}

La leishmaniasis es un problema de salud pública, encontrándose dentro del grupo de las enfermedades desatendidas, debido a que afecta principalmente a población de bajos recursos económicos de países en vías de desarrollo ${ }^{1,12}$. Se ha reportado que, en el Departamento de Huánuco, Perú cuenta con diversas especies de leishmania causantes de la enfermedad; entre las más frecuentes tenemos a $L$. (V.) braziliensis, $L$. (V.) peruviana, $L$. (V.) guyanensis y $L .(V$.$) lainsoni; así como una nueva especie$ híbrida de $L$. (V.) braziliensis/L. (V.) peruviana ${ }^{13,14}$. El principal vector identificado fue la especie Lutzomyia tejadai seguida de Lutzomia fischeri ${ }^{14}$. Debido a las altas tasas de incidencia de leishmaniasis tegumentaria en este departamento durante el siglo pasado ${ }^{10,11}$, investigaciones han reportado que las tasas de leishmaniasis canina (8-45\%) juegan un importante rol en la transmisión peridoméstica de la enfermedad ${ }^{15,16}$

Nuestro estudio identificó que la forma clínica cutánea de la enfermedad es la más frecuente; sin embargo, la forma muco-cutánea ha venido presentando una incidencia constante durante los últimos años, probablemente debido a la presencia de la especie híbrida entre $L$. brazilensis/ $L$. peruviana, cuyo vector identificado fue $L$. tejadai ${ }^{14}$. Esta nueva especie circulante fue encontrada únicamente en esta zona del país y su particular comportamiento epidemiológico es debido, al parecer, por la caracterización genética entre Leishmania andina (peruviana) y selvática (brazilensis); esta última traída posiblemente por habitantes que retornaron de la región amazónica durante la época de la lucha armada de grupos guerrilleros en el Perú ${ }^{13}$. Durante la década pasada, en esta región se reportaron tasas de incidencia anual que bordearon los 413/100.000 habts., siendo mayor a países hiperendémicos como Argelia (202,6/100.000 habts.), Afganistán $(226,2 / 100.000 \text { habts.) e Irán (113,3/100.000 habts. })^{7}$. No obstante, ahora observamos un patrón decreciente en las tasas de incidencia anual de la enfermedad, que en el año 2017 llegó a una cifra de 46/100.000 habts., siendo considerado como un riesgo de infección moderada. En la actualidad países como Siria, Afganistán, Pakistán y Brasil son los que reportan tasas de prevalencias más altos a nivel mundial, colocando al Perú en el puesto número 6 con una tasa de 13,4/100.000 habts $^{8,17}$.

La leishmaniasis registró una mayor prevalencia en el género masculino; asimismo se identificó que el género femenino encabezó la población afectada por la presentación clínica cutánea mientras que el masculino en la forma muco-cutánea. Se ha descrito que en la forma cutánea existe una relación entre la población masculina y femenina de 3:2 y en la muco-cutánea de 3:1 respectivamente; asimismo, los pacientes varones presentan una mayor afectación de la enfermedad que en las mujeres (70 vs $30 \%$; RR $=2,77$; IC 95\%: 1,77-4,33), conllevando a tasas superiores de incidencia anual en el primer grupo por encima del segundo $(0,5 / 100.000$ habts. vs $0,2 / 100.000$ habts. $)^{18-21}$. Nosotros encontramos una elevada prevalencia de la enfermedad en pacientes entre 1 y 10 años seguido por edades de 11 a 20 años; estos hallazgos concuerdan con diversas investigaciones donde manifiestan que los mayores índices de la enfermedad (18-39\%) se encuentran en edades entre 1 a 20 años ${ }^{19,22-26}$. Además, se ha reportado elevadas tasas de incidencia de leishmaniasis en población pediátrica (1,53/100.000 habts.), siendo un indicador de la transmisión doméstica de la enfermedad ${ }^{19,27,28}$. Por otro lado, en países como África (Burkina Faso), Argentina, México, Brasil (Londrina y Jequitinhonha) y Perú (Loreto y Madre de Dios), se ha descrito que las poblaciones de pacientes entre 20 a 60 años representan los mayores índices (35-55\%) de infección ${ }^{21,22,29-33}$.

En la zona urbana de toda la provincia de Ambo se reportaron cifras sobre $35 \%$ para ambas formas clínicas de la enfermedad. Esto concuerda con estudios donde describen que 25 a $80 \%$ de la población infectada proviene frecuentemente de zona urbana ${ }^{23,25,30}$; opuestamente, en la India se identificó que las poblaciones pertenecientes a zonas rurales representan las mayores tasas de infección, planteando una relación de 4:1 entre esta zona y la urbana ${ }^{25}$. Se encontró una elevada prevalencia de la forma clínica cutánea en comparación con la forma mucocutánea en el área urbana del distrito de Ambo (43,1 vs $38,2 \%$ ), mientras que en el distrito de Huacar, cuya población es de carácter rural, se evidenció una mayor incidencia de la forma muco-cutánea en comparación con la cutánea ( 26,8 vs $22,2 \%)$; estas diferencias son poco usuales debido a la cercanía geográfica $(<10 \mathrm{~km})$ y el similar clima templado de ambas zonas. Es importante el desarrollo de investigaciones que traten de explicar estos hallazgos entre los diversos distritos de la Provincia de Ambo.

El género masculino es el principal afectado de la enfermedad debido a una mayor exposición al vector por las labores a campo abierto ${ }^{34}$. Nuestros hallazgos corroboran esta afirmación, debido que el ámbito laboral de la agricultura y la ganadería representaron uno de los elevados índices de enfermedad para ambas formas clínicas. Las tasas más elevadas de prevalencia se concentraron en pacientes desempleados; se ha descrito que los habitantes nativos de las zonas endémicas, principalmente población económicamente no activa como el caso de las amas de casa y estudiantes, presentan las mayores tasas de infección $(31,2 \%)^{19,22,25,29}$ demostrando la gran capacidad de adaptación del vector a los ambientes domiciliarios y peri-domiciliarios ${ }^{34}$. Dentro del grupo de habitantes foráneos, los trabajadores y profesionales representaron la mayor incidencia $(56,6 \%)$ de esta infección ${ }^{28}$. 
Más de 50\% de las lesiones afectaron la región facial, seguido de los miembros superiores y el tronco; estos hallazgos son semejantes a los descritos en un estudio en Turquía, donde la región facial fue la principal zona afectada, seguida de los miembros superiores e inferiores $(57,3 \text { vs } 42,4 \%)^{26}$. Estudios realizados en Argentina, Ecuador y Brasil, concluyeron que las zonas corporales más afectadas son los miembros superiores e inferiores (39-71\%) seguidos de la cara y el tronco (15-20\%) $)^{24,31,32}$. Esta particular distribución de las lesiones identificadas en nuestro estudio podría deberse al clima templado, ocasionando que las personas vistan atuendos que los protejan, siendo la región de la cara, la única zona que se encuentra descubierta y expuesta a los vectores de la enfermedad.

Entre los principales métodos diagnósticos, se identificó que el más usado fue la microscopía seguido de la IFI ( 81,5 vs $18,5 \%)$; datos similares se obtuvieron en reportes de zonas endémicas de la India, México, Brasil y Perú, donde las pruebas de diagnóstico parasitológico directo (microscopía) fueron las de principal uso (47 a $60 \%$ ), luego las pruebas serológicas (IFI) y en menor medida la reacción de polimerasa en cadena (RPC) $)^{21,22,25,29}$. El uso de la microscopía directa como principal método diagnóstico, se ha masificado a lo largo de las zonas endémicas debido a la facilidad de uso y rapidez del diagnóstico, especialmente en áreas rurales, frente a la poca disponibilidad de los test serológicos y/o moleculares $^{22,25,29}$. Un estudio peruano reportó que la microscopía directa está sujeta a elevados diagnósticos inexactos (31\%; OR = 1,93; IC 95\%; 1,06-3,53) conllevando en ocasiones a terapias innecesarias y cursos prolongados de la enfermedad ${ }^{29}$.

Se obtuvieron tasas de curación superiores en la forma clínica muco-cutánea frente a la cutánea (64,5 vs 56,9\%). Estos hallazgos concuerdan por los descritos por Da Silva y Pontello, quienes reportaron tasas de curación que bordeaban el 44 a 49\% para ambas forma clínicas y además junto a Matos y Falqueto, manifestaron que $23,3 \%$ de los pacientes con leishmaniasis requirieron al menos 3-5 ciclos de terapia antimonial para obtener cifras de curación superiores a $90 \%{ }^{18,22,32,34}$. Un estudio llevado a cabo en el Perú, identificó que existe una tasa de $24,4 \%$ de fracaso terapéutico para leishmaniasis en su forma clínica cutánea, la edad del paciente, residencia habitual, curso clínico de la enfermedad e infección por especies de $L$. (V.) peruviana y $L$. (V.) braziliensis son los principales factores que contribuyeron a la persistencia de la enfermedad ${ }^{9}$. En nuestro estudio, $25 \%$ de la población infectada persistió con la enfermedad a pesar de haber finalizado el tratamiento. Pese a la problemática que ha generado los fallos del tratamiento para la leishmaniasis cutánea y muco-cutánea, la comunidad científica aún concentra sus esfuerzos en la presentación clínica visceral; esto debido a su curso clínico tórpido y creciente mortalidad $^{12}$.

La leishmaniasis implica un importante problema de salud mundial, debido al aumento de la infección y las ineficientes políticas de salud pública. Se logrará el control de la enfermedad con adecuadas estrategias que incluyan un diagnóstico, tratamiento oportuno y conocimiento sobre el comportamiento y la dinámica de la enfermedad.

Agradecimientos. A la Red de Salud Ambo y a la Oficina General de Epidemiología de la Dirección Regional de Salud del Departamento de Huánuco, Perú, por la ayuda técnica durante el desarrollo de la presente investigación.

\section{Resumen}

Introducción: La leishmaniasis es una enfermedad infecciosa que presenta una elevada prevalencia e incidencia mundial, causando alrededor de 70.000 muertes anuales. Objetivo: Determinar el perfil clínico y epidemiológico de los casos de leishmaniasis tegumentaria en la Provincia de Ambo, Departamento de Huánuco, Perú, durante el período: años 2000 a 2017. Métodos: Se realizó un estudio descriptivo, observacional, retrospectivo y transversal de base poblacional. Resultados: Se identificaron 1.312 pacientes confirmados de leishmaniasis tegumentaria. La forma clínica cutánea tuvo una mayor prevalencia en comparación a la muco-cutánea $(83,2$ vs $16,7 \%)$. La infección en el género masculino tuvo mayor predominio frente al femenino (53,3 vs 46,6\%). La mayor tasa de incidencia anual reportada fue de 412,67/100.000 habts. durante el año 2000 mientras que la menor registrada fue en el 2015 con 24,45/100.000 habts. El Distrito de Ambo fue el más afectado con la forma clínica cutánea $(43,1 \%)$ y la muco-cutánea $(38,2 \%)$ de la enfermedad. El principal método diagnóstico para ambas formas clínicas fue el examen microscópico directo $(82,0$ vs $81,0 \%)$ y la terapia más utilizada fue con estibogluconato de sodio ( 85,6 vs $78,6 \%$ ). Se reportó falla al tratamiento de $25 \%$ para ambas presentaciones clínicas. Conclusiones: Las condiciones eco-ambientales de esta zona del Perú han permitido el establecimiento de la leishmaniasis tegumentaria; mostrando tendencias similares a las comunicadas en la literatura mundial. Sin embargo, se necesitan más investigaciones que expliquen el comportamiento epidemiológico de esta enfermedad y permitan crear modelos de predicción epidemiológica para un control adecuado y oportuno. 


\section{Referencias bibliográficas}

1.- Karimkhani C, Wanga V, Naghavi P, Dellavalle R P, Naghavi M. Global burden of cutaneous leishmaniasis. Lancet Infect Dis 2017; 17 (3): 264. doi: 10.1016/S14733099(16)30217-1.

2.- Al-Salem W, Herricks J R, Hotez P J. A review of visceral leishmaniasis during the conflict in South Sudan and the consequences for East African countries. Parasit Vectors. 2016; 9 (1): 460. doi: 10.1186/s13071-016-1743-7.

3.- Mhaidi I, El Kacem S, Kbaich M A, El Hamouchi A, Sarih M, Akarid K, et al. Molecular identification of Leishmania infection in the most relevant sand fly species and in patient skin samples from a cutaneous leishmaniasis focus, in Morocco. PLoS Negl Trop Dis. 2018; 12(3): e0006315. doi: 10.1371/ journal.pntd.0006315.

4.- Mathers C D, Ezzati M, López A D. Measuring the burden of neglected tropical diseases: the global burden of disease framework. PLoS Negl Trop Dis. 2007; 1(2): e114. doi: 10.1371/ journal.pntd.0000114.

5.- Torres-Guerrero E, Quintanilla-Cedillo MR, Ruiz-Esmenjaud J, Arenas R. Leishmaniasis: a review. F1000Res. 2017; 6: 750. doi: 10.12688/ f1000research.11120.1.

6.- Pigott D M, Bhatt S, Golding N, Duda K A, Battle K E, Brady O J, et al. Global distribution maps of the leishmaniases. Elife. 2014; 3. doi: 10.7554/ELIFE.02851.

7.- Alvar J, Velez I D, Bern C, Herrero M, Desjeux $\mathrm{P}$, Cano J, et al. Leishmaniasis worldwide and global estimates of its incidence PLoS One. 2012; 7(5): e35671. https://doi.org/10.1371/ journal.pone. 0035671 .

8.- Centro Nacional de Epidemiologia, Prevención y Control de Enfermedades MINSA. [Internet] [citado el 4 de julio de 2018]. Sala situacional para el Análisis de Situación de Salud en Enfermedades Metaxénicas: Leishmaniasis Ministerio de Salud del Perú 2018. Disponible en: http://www.dge.gob.pe/portal/index. php?option $=$ com_content $\&$ view $=$ article $\&$ id $=14$ \&Itemid $=121$.

9.- Llanos-Cuentas A, Tulliano G, Araujo-Castillo R, Miranda-Verastegui C, SantamariaCastrellon G, Ramírez L, et al. Clinical and parasite species risk factors for pentavalent antimonial treatment failure in cutaneous leishmaniasis in Peru. Clin Infect Dis 2008; 46 (2): 223-31. doi: 10.1086/524042.

10.- Tejada A, Tejada O, Zorrilla V. Leishmaniasis tegumentaria en Ambo, Huánuco. Rev Per Med Trop UNMSM 2001; 1 (1): 21-8. http://sisbib. unmsm.edu.pe/BVRevistas/SPEIT/2001_n1/ Articulo_Original/pag_22-25.htm.

11.- Programa de Control de Malaria y Otras Enfermedades Metaxénicas MINSA. [Internet] [citado el 4 de julio de 2018].
Normas y Procedimientos para el Control de la Leishmaniasis en el Perú. 1993: 16-20. Disponible en: http://bvs.minsa.gob.pe/local/ dgsp/248_nor14.pdf.

12.- Carvalho E M, Llanos-Cuentas A, Romero G A S. Mucosal leishmaniasis: urgent need for more research. Rev Soc Bras Med Trop 2018; 51 (1): 120-1. http://dx.doi.org/10.1590/0037-86820463-2017.

13.- Koarashi Y, Cáceres A G, Saca F M Z, Flores E E P, Trujillo A C, Alvares J L A, et al. Identification of causative Leishmania species in Giemsa-stained smears prepared from patients with cutaneous leishmaniasis in Peru using PCR-RFLP. Acta Trop 2016; 158: 83-7. doi: 10.1016/j.actatropica.2016.02.024.

14.- Kato H, Cáceres AG, Hashiguchi Y. First evidence of a hybrid of Leishmania (Viannia) braziliensis/L.(V.) peruviana DNA detected from the phlebotomine sand fly Lutzomyia tejadai in Peru. PLoS Negl Trop Dis 2016; 10 (1): e0004336. https://doi.org/10.1371/journal. pntd.0004336.

15.- Reithinger R, Dujardin J-C, Louzir H, Pirmez C, Alexander B, Brooker S. Cutaneous leishmaniasis. Lancet Infect Dis. 2007; 7(9): 581-96. doi: 10.1016/S1473-3099(07)70209-8.

16.- Reithinger R, Espinoza J C, Davies C R. The transmission dynamics of canine American cutaneous leishmaniasis in Huánuco, Peru. Am J Trop Med Hyg 2003; 69 (5): 473-80. PMID: 14695083.

17.- World Health Organization. Number of cases of cutaneous leishmaniasis reported Data by country. [Internet] [Actualizado el $15 \mathrm{de}$ septiembre de 2017, citado el 4 de julio de 2018]. Disponible en: http://apps.who.int/gho/ data/view.main.NTDLEISHCNUMv.

18.- Falqueto A, Sessa P, Ferreira A, Vieira VP, Santos CB, Varejão JB, et al. Epidemiological and clinical features of Leishmania (Viannia) braziliensis American cutaneous and mucocutaneous leishmaniasis in the State of Espírito Santo, Brazil. Mem Inst Oswaldo Cruz 2003; 98 (8): 1003-10. doi: 10.1590/s007402762003000800004.

19.- Riera C, Napp S, Manzanares S. Epidemiología de la leishmaniasis humana en la ciudad de Barcelona (1997-2014). Rev Enf Emerg 2016; 15: 68-76. http://enfermedadesemergentes.com/ revistas/enf-emergentes_2-2016.pdf.

20.- Kassiri H, Sharifinia N, Jalilian M, Shemshad K. Epidemiological aspects of cutaneous leishmaniasis in Ilam province, west of Iran (2000-2007). Asian Pac J Trop Dis 2012; 2: S382-S6. https://doi.org/10.1016/S22221808(12)60186-8.

21.- Javier S Á E, Janett C L, Renato Z C I. Perfil epidemiológico de la leishmaniasis: una enfermedad olvidada en México. Enf Inf Microbial 2014; 34 (1): 31. https://www. medigraphic.com/pdfs/micro/ei-2014/ei141e.pdf.
22.- Nunes W d S, Araújo S R, Calheiros C M L. Epidemiological profile of leishmaniasis at a reference service in the state of Alagoas, Brazil, from January 2000 to September 2008. Braz J Infect Dis 2010; 14 (4): 342-5. http://dx.doi. org/10.1590/S1413-86702010000400005.

23.- Adel A, Boughoufalah A, Saegerman C, De Deken R, Bouchene Z, Soukehal A, et al. Epidemiology of visceral leishmaniasis in Algeria: an update. PLoS One 2014; 9 (6): e99207. https://doi.org/10.1371/journal. pone.0099207.

24.- Gómez E A, Kato H, Torres-Romero E X, Velez L N, Villegas N V, Martillo V P, et al. Leishmaniasis caused by Leishmania (Viannia) guyanensis in north-central Pacific region of Ecuador: a clinico-epidemiological feature. Acta Trop 2018. doi: 10.1016/j. actatropica.2018.05.016.

25.- Aara N, Khandelwal K, Bumb R A, Mehta R D, Ghiya B C, Jakhar R, et al. Clinico-epidemiologic study of cutaneous leishmaniasis in Bikaner, Rajasthan, India. Am J Trop Med Hy 2013; 89 (1): 111-5. doi: 10.4269/ajtmh.12-0558.

26.- Gurel M S, Ulukanligil M, Ozbilge H. Cutaneous leishmaniasis in Sanliurfa: epidemiologic and clinical features of the last four years (1997-2000). Int J Dermatol. 2002; 41 (1): 32-7. doi: 10.1046/j.00119059.2001.01396.x.

27.- Blanco V M, Cossio A, Martínez J D, Saravia $\mathrm{N}$ G. Clinical and epidemiologic profile of cutaneous leishmaniasis in Colombian children: considerations for local treatment. Am J Trop Med Hyg 2013; 89 (2): 359-64. doi: 10.4269/ ajtmh.12-0784.

28.- Wang J-Y, Cui G, Chen H-T, Zhou X-N, Gao C-H, Yang Y-T. Current epidemiological profile and features of visceral leishmaniasis in People's Republic of China. Parasit Vectors 2012; 5 (1): 31. https://doi.org/10.1186/17563305-5-31.

29.- Lamm R, Alves C, Perrotta G, Murphy M, Messina C, Sánchez JF, et al. Prevalence of and factors associated with negative microscopic diagnosis of cutaneous leishmaniasis in rural Peru. Am J Trop Med Hyg 2018. doi: 10.4269/ ajtmh.17-0909.

30.- Soares M, Pedrosa-Silva A L, Rocha S L, Lima N L, Verli F D, Ferreira Fde O, et al. Epidemiological profile of individuals with American cutaneous leishmaniasis in Jequitinhonha Valley, Brazil. East Mediterr Health J. 2014; 19: S81-8. PMID: 24995766.

31.- Bustos M F G, González-Prieto G, Ramos F, Mora M C, Hashiguchi Y, Parodi C, et al. Clinical and epidemiological features of leishmaniasis in northwestern-Argentina through a retrospective analysis of recent cases. Acta Trop. 2016; 154: 125-32. doi: 10.1016/j. actatropica.2015.11.008. 
32.- Pontello Junior R, Gon A d S, Ogama

A. American cutaneous leishmaniasis: epidemiological profile of patients treated in Londrina from 1998 to 2009. Ann Bras Dermatol 2013; 88: 748-53. doi: 10.1590/ abd1806-4841.20132168.

33.- Bamba S, Gouba A, Drabo M K, Nezien D,
Bougoum M, Guiguemdé T R.

Epidemiological profile of cutaneous leishmaniasis: Retrospective analysis of 7444 cases reported from 1999 to 2005 at Ouagadougou, Burkina Faso. Pan Afr Med J 2013; 14 (1). doi: 10.11604/ pamj.2013.14.108.1140.
34.- Rocha T J M, Barbosa A C A, Santana E P C, Calheiros C M L. Aspectos epidemiológicos dos casos humanos confirmados de leishmaniose tegumentar americana no Estado de Alagoas, Brasil. Rev Pan-Amaz Saud 2015; 6: 49-54. http://dx.doi.org/10.5123/S217662232015000400007 . 